\title{
Criminologie
}

\section{L'Association Mondiale des Amis de l'Enfance et les problèmes de la structuration du bénévolat}

\section{Alice Parizeau}

Volume 11, numéro 1, 1978

Centres d'accueil du Québec : accréditation et évaluation

URI : https://id.erudit.org/iderudit/017084ar

DOI : https://doi.org/10.7202/017084ar

Aller au sommaire du numéro

Éditeur(s)

Les Presses de l'Université de Montréal

ISSN

0316-0041 (imprimé)

1492-1367 (numérique)

Découvrir la revue

Citer ce compte rendu

Parizeau, A. (1978). Compte rendu de [L'Association Mondiale des Amis de l'Enfance et les problèmes de la structuration du bénévolat]. Criminologie, 11(1), 75-79. https://doi.org/10.7202/017084ar d'utilisation que vous pouvez consulter en ligne. 
L'ASSOCIATION MONDIALE DES AMIS DE L'ENFANCE ET LES PROBLÈMES DE LA STRUCTURATION DU BENEVOLAT

Alice Parizeau

Depuis 1970, on constate autant aux États-Unis qu'au Canada, un développement de législations concernant la protection de l'enfance. En effet, dans les divers états américains comme dans certaines provinces canadiennes, les législateurs ont jugé indispensable de compléter les dispositions déjà en vigueur et de renforcir l'intervention de la collectivité en vue d'aider et de prendre en charge des enfants maltraités physiquement, comme ceux qui ne reçoivent pas dans leur famille de soins adéquats. Parallèlement, on constate une multiplication de services sociaux ayant pour mission le dépistage et l'assistance auprès des familles et des enfants désavantagés.

Cela signifie que les législations s'accompagnent de règlements administratifs et de normes. Pour compenser les déficiences du cadre familial, la société est obligée, en quelque sorte, d'élaborer des modèles d'action d'autant plus complexes qu'il convient de tenir compte des droits des parents et de la reconnaissance de l'autorité parentale qui freine l'intervention préventive et s'exerce parfois au détriment des droits des enfants. L'action sociale est paralysée par le conflit qui existe entre le principe de la liberté individuelle et le devoir de la collectivité de protéger ses futurs citoyens. La complexité des situations individuelles rend, en outre, le travail des services sociaux d'autant plus critiquable que ses résultats ne peuvent être évalués qu'à très long terme.

Les coûts des services augmentent constamment et atteignent des montants de $\$ 10000^{1}$ par an et par enfant quand il s'agit de placement dans une institution spécialisée et variables pour ceux qui sont pris quotidiennement en charge pendant les heures ouvrables. L'augmentation du pourcentage d'enfants ayant besoin d'aide étant liée, semble-t-il, à l'accroissement du taux de

1. Québec, ministère des Affaires sociales (Président : M. G. Batshaw). La Clientele des centres d'accueil de transition et de réadaptation du Québec, par Marc LeBlanc. Annexe 6 du rapport du Comité d'étude sur la réadaptation des enfants et adolescents placés en centre d'accueil. Québec, ministère des Affaires sociales, 1975. 
séparations, de divorces, des échecs scolaires, d'absentéisme et d'abandon précoce des études, la tendance apparaît comme irréversible.

Par ailleurs, en Amérique du Nord, l'intervention de la collectivité, limitée par le passé à la protection de l'enfance malheureuse, maltraitée et délinquante, s'élargit à l'ensemble des enfants d'un certain âge dont les familles réclament des modalités de prise en charge pendant les heures ouvrables. Compte tenu des augmentations de taxes qu'entraînent tous ces services qui sont totalement ou partiellement subventionnés, ils doivent être organisés conformément à la demande et aux besoins des contribuables. Plus nombreux dans les quartiers désavantagés et moins répandus ailleurs, ils parviennent à satisfaire parfois les exigences immédiates des familles monoparentales et celles où les deux parents travaillent, mais il n'est pas certain, pour autant qu'ils répondent vraiment aux besoins des enfants.

En somme, au Canada, comme aux Etats-Unis, le développement brusque, comparativement à celui de certains pays européens, des services à l'enfance, occasionne une charge financière croissante, mais l'évaluation des résultats obtenus laisse à désirer.

Où se situent dès lors les droits de l'enfance à recevoir des soins et une protection adéquate? Qui a la charge de reconnaître et de défendre ces droits ?

Il est difficile, en effet, de concevoir un appareil administratif, ayant un personnel important et professionnalisé, tant bien que mal d'ailleurs, susceptible de reconnaître ses lacunes à la suite des protestations de ses jeunes clients. Il est tout aussi malaisé d'espérer que les normes législatives, aussi parfaites puissent-elles être, sont en mesure d'assurer un cadre suffisamment rigide pour éliminer les abus et, à plus forte raison, les lacunes du personnel chargé de les appliquer.

C'est ainsi qu'au Québec, par exemple, la nouvelle loi de protection de l'enfance, présentée par le ministre Pierre Marois est fort intéressante, mais il est évident que les résultats qu'elle permettra d'obtenir dépendent essentiellement de la façon selon laquelle elle sera mise en pratique.

Et dans un domaine aussi complexe que celui de l'aide et de la protection de l'enfance, l'administration ne peut dans aucun cas être à la fois l'exécutant et l'évaluateur de sa propre action... 
Il en ressort qu'une implication de la collectivité demeure indispensable et cela dans n'importe quel contexte socio-économique. Implication au niveau des individus, prêts à travailler gratuitement, comme des groupes constitués spontanément, tels entre autres, des mouvements communautaires de quartier. Toutefois, le bénévolat d'aujourd'hui ne ressemble guère à celui d'hier. La « dame-patronnesse », personne généralement indépendante financièrement, qui s'occupait d'une ou de plusieurs familles et qui, en échange, était respectée pour son dévouement, est considérée désormais comme un individu qui entre en concurrence avec des travailleurs sociaux, les gêne dans leur action, ou pis encore va à l'encontre des intérêts des professionnels en période de grève. Le bénévolat, individualisé par définition, qu'on a connu dans le passé, est actuellement un phénomène dépassé. Celles et ceux qui veulent s'impliquer gratuitement pour aider les autres, sont obligés d'accepter des cadres structurés, c'est-à-dire faire partie des associations et des groupements. Singulièrement, quand il s'agit de la reconnaissance des droits des enfants, où la possibilité d'entrer en conflit avec des services sociaux, judiciaires et scolaires est plus élevée que quand il s'agit des droits des adultes, les associations bénévoles doivent être non seulement locales, mais encore pouvoir compter sur un appui qu'elles ne peuvent obtenir qu'à la faveur d'un regroupement plus important.

A l'instar des organismes, telle l'Amnistie internationale qui a pour vocation de protéger les prisonniers politiques, il faut probablement chercher à constituer des organes supra-nationaux chargés de défendre les droits des enfants qui eux aussi sont en quelque sorte des «prisonniers » des contextes socio-culturels et éducatifs dans lesquels ils sont appelés à vivre et à évoluer et dont chacun génère des tabous imposés par des adultes.

L'Association mondiale des amis de l'enfance, créée il y a sept ans à Monaco, constitue, à cet égard, un organe de regroupement privilégié. Sur le plan administratif, la société a adopté des normes largement inspirées de celles de la Croix-Rouge et elle a obtenu jusqu'à présent l'adhésion de la Belgique, de l'Allemagne Fédérale, de la France, de Monaco et du Liban. On peut y voir la preuve qu'il s'agit d'une association viable, c'est-à-dire établie sur des bases suffisamment solides pour élargir à l'avenir son champ d'action. 
En ce qui a trait à ses objectifs, ils consistent à « soutenir et à promouvoir toutes organisations et entreprises se proposant d'assurer le bien-être physique, moral et spirituel de l'enfance dans le monde ».

Sur le plan de l'action concrète, l'Association a défendu tout aussi bien les droits des enfants des pays sous-développés que des droits de ceux qui ont vécu la guerre au Vietnam, et sa vocation n'est pas limitée aux interventions faites à l'occasion des crises et des cataclysmes.

L'Association mondiale des amis de l'enfance (Amade ${ }^{2}$ ), constitue, à cet égard, un des moyens de regroupement du bénévolat, local, régional, ou national.

Certes, l'idée d'un organisme défini comme celui des \& Amis de l'enfance », n'est pas nouvelle, ce qui est particulier, c'est que l'Association se veut similaire aux structures qui se préoccupent de défendre les droits des adultes, dont, entre autres, la CroixRouge.

Est-ce un symptôme particulier à notre époque, que cette défense des droits des enfants considérés à certains égards comme des « consommateurs $\gg$ des biens et des services, plutôt que comme des citoyens de demain dont dépend l'avenir collectif ?

C'est la question qu'on ne peut s'empêcher de poser en constatant une sorte de regain de vitalité du bénévolat qui apparait à travers les mouvements communautaires locaux, comme sur le plan international dans le cadre des associations qui ressemblent étrangement à celles qui sont apparues, en ce qui a trait aux adultes, au début du XXe siècle.

Tout se passe en somme, comme si à l'approche marquée par le respect absolu de l'autorité parentale devait succéder la tendance visant à préciser, à défendre et à imposer, face à la société adulte, une certaine autonomie de l'enfance. Autonomie découlant du droit de l'enfant d'exprimer ses besoins personnellement, et non seulement par l'entremise de sa famille ou de services sociaux qui ont la charge de l'aider, mais qui lui imposent par le fait même un cadre de vie défini par l'autorité administrative comme une norme adaptable à tous les jeunes du même

2. L'Association mondiale des amis de l'enfance, (Amade), 16, Bd de Suisse, Monte-Carlo. 
âge, sans considération aucune pour les aspirations particulières de certains individus et de certains groupes.

Au-delà des services formels apparaissent dès lors, des organismes informels, et bénévoles, locaux, nationaux et supra nationaux, telle l'Association mondiale des amis de l'enfance, qui sont appelés à se développer parce qu'ils répondent au besoin, devenu urgent à notre époque, de défendre les droits des enfants au même titre que c'est déjà le cas pour les droits de l'homme. 